\title{
Existence of Multiple Nontrivial Solutions for a Strongly Indefinite Schrödinger-Poisson System
}

\author{
Shaowei Chen and Liqin Xiao \\ School of Mathematical Sciences, Huaqiao University, Quanzhou 362021, China \\ Correspondence should be addressed to Shaowei Chen; chensw@amss.ac.cn \\ Received 27 November 2013; Accepted 4 January 2014; Published 19 February 2014 \\ Academic Editor: Chun-Lei Tang
}

Copyright (c) 2014 S. Chen and L. Xiao. This is an open access article distributed under the Creative Commons Attribution License, which permits unrestricted use, distribution, and reproduction in any medium, provided the original work is properly cited.

We consider a Schrödinger-Poisson system in $\mathbb{R}^{3}$ with a strongly indefinite potential and a general nonlinearity. Its variational functional does not satisfy the global linking geometry. We obtain a nontrivial solution and, in case of odd nonlinearity, infinitely many solutions using the local linking and improved fountain theorems, respectively.

\section{Introduction and Statement of Results}

In this paper, we consider the Schrödinger-Poisson system:

$$
\begin{gathered}
-\Delta u+V(x) u+K_{1}(x) \phi u=Q(x) f(u), \quad \text { in } \mathbb{R}^{3}, \\
-\Delta \phi=K_{2}(x) u^{2}, \quad \text { in } \mathbb{R}^{3} .
\end{gathered}
$$

For $V, K_{1}, K_{2}, Q$, and $f$, we assume the following.

(v) $V \in L_{\text {loc }}^{q}\left(\mathbb{R}^{3}\right)$ and $V^{-}=\min \{-V, 0\} \in L^{\infty}\left(\mathbb{R}^{3}\right)+$ $L^{q}\left(\mathbb{R}^{3}\right)$ for some $q \in[2, \infty)$. This assumption ensures that the Schrödinger operator $S=-\Delta+V$ is selfadjoint and semibounded on $L^{2}\left(\mathbb{R}^{3}\right)$ (see Theorem A.2.7 in [1]). $\sigma(S)$ denotes the spectrum of $S$. We assume that 0 lies in a gap of $\sigma(S)$; that is, there exist $\alpha, \beta>0$ such that

$$
\sigma(S) \cap(-\alpha, \beta)=\emptyset .
$$

(k) $Q, K_{i} \in C\left(\mathbb{R}^{3}\right)$ satisfy $Q(x)>0, K_{i}(x)>0$ for all $x$,

$$
\lim _{|x| \rightarrow \infty} Q(x)=0, \quad \lim _{|x| \rightarrow \infty} K_{i}(x)=0, \quad i=1,2,
$$

and there exists $p \in(4,6)$ such that

$$
\int_{\mathbb{R}^{3}} \frac{K_{i}^{6 p /(5 p-12)}(x)}{Q^{12 /(5 p-12)}(x)} d x<\infty, \quad i=1,2 .
$$

$\left(\mathbf{f}_{1}\right) f \in C(\mathbb{R})$ and there exists $C>0$ such that for all $t \in \mathbb{R}$

$$
|f(t)| \leq C\left(|t|+|t|^{p-1}\right) .
$$

$\left(\mathbf{f}_{2}\right) f(t)=o(t)$ as $t \rightarrow 0$.

$\left(\mathbf{f}_{3}\right)$ Let

$$
F(t)=\int_{0}^{t} f(\tau) d \tau
$$

We assume that there exists $A>0$ such that for all $t \in \mathbb{R}$

$$
\widetilde{F}(t):=t f(t)-2 F(t) \geq A|t|^{p} .
$$

$\left(\mathbf{f}_{4}\right) f(-t)=-f(t)$ for all $t \in \mathbb{R}$.

Remark 1. Note that

$$
\left(\frac{F(t)}{t^{2}}\right)^{\prime}=\frac{t f(t)-2 F(t)}{t^{3}} .
$$

This together with (7) implies that there exists $C_{1}>0$ such that for all $t \in \mathbb{R}$,

$$
F(t) \geq C_{1}|t|^{p} .
$$

Our main results are as follows. 
Theorem 2. Suppose that $(\mathbf{v}),(\mathbf{k})$, and $\left(\mathbf{f}_{\mathbf{1}}\right)-\left(\mathbf{f}_{\mathbf{3}}\right)$ are satisfied, then the problem (1) has a nontrivial solution.

Theorem 3. Suppose that $(\mathbf{v}),(\mathbf{k})$, and $\left(\mathbf{f}_{\mathbf{1}}\right)-\left(\mathbf{f}_{\mathbf{4}}\right)$ are satisfied, then the problem (1) has infinitely many solutions.

Problem (1) arises in quantum mechanics and is related to the study of the nonlinear Schrödinger equation for a particle in an electromagnetic field or the Hartree-Fock equation. For a more detailed physical background of the SchrödingerPoisson system, readers can refer to $[2,3]$ and the references therein.

This system has attracted considerable research attention in the recent decade, and it has been studied widely by using the modern variational method and critical point theory under various assumptions. However, many mathematical studies have been devoted to the case $\inf _{\mathbb{R}^{3}} V>0$. In this case, there are many results on the existence, nonexistence, or multiplicity of solutions for (1). One can refer to [2-20].

There are very few studies devoted to (1) under the assumption that $-\Delta+V$ has a nontrivial negative eigenspace compared to the case $\inf _{\mathbb{R}^{3}} V>0$. In a recent paper [21], Chen and Liu studied the problem under the assumption on $V$ that

$\left(\mathbf{v}^{\prime}\right) V \in C\left(\mathbb{R}^{3}\right)$ is bounded from below and $\mu\left(V^{-1}(-\infty\right.$, $M])<\infty$ for every $M>0$, where $\mu$ is the Lebesgue measure on $\mathbb{R}^{3}$. Moreover, the operator $-\Delta+V$ has negative eigenvalues.

They verified the existence of multiple solutions of (1) under this assumption on $V$ and under certain 4-superlinear conditions on $f$. Our assumptions on $V$, which are different from $\left(\mathbf{v}^{\prime}\right)$, allow an infinitely dimensional negative eigenspace of $-\Delta+V$. This causes some difficulties. For example, it makes the verification of the compactness conditions a more delicate problem. In addition, when we search for infinitely many solutions of (1) for the case where $f$ is odd, the classical fountain theorem of Bartsch (see [22] or [23]) cannot be applied. Fortunately, this difficulty can be overcome using a recently improved fountain theorem of Batkama and Colin [24]. To the best of our knowledge, the Schrödinger-Poisson equation with a strongly indefinite linear part has never been studied. Besides the difficulties caused by the strongly indefinite linear part, the functional related to (1) (see Section 2) involves a nonlocal term and it makes the functional not satisfy the global linking structure. To overcome this difficulty and obtain a nontrivial solution of (1), we use the local linking method (see [25]).

Throughout this paper, we denote the strong and the weak convergence by $\rightarrow$ and $\rightarrow$, respectively. $L^{p}\left(\mathbb{R}^{3}\right)$ denotes the standard Lebesgue space with norm $\|u\|_{L^{p}}=$ $\left(\int_{\mathbb{R}^{3}}|u|^{p} d x\right)^{1 / p}$. For $k \in \mathbb{N}, H^{k}\left(\mathbb{R}^{3}\right)$ denotes the standard Sobolev space with norm $\|u\|_{H^{k}}=\left(\int_{\mathbb{R}^{3}} \sum_{|\gamma| \leq k}\left|\partial^{\gamma} u\right|^{2} d x\right)^{1 / 2}$. For a Banach space $E$, we denote the dual space of $E$ by $E^{*}$, and the norm of $E^{*}$ is denoted by $\|\cdot\|_{E^{*}}$.

\section{Proof of Theorem 2}

Assume that (v) holds and let $S=-\Delta+V$ be the self-adjoint operator acting on $L^{2}\left(\mathbb{R}^{3}\right)$ with domain $D(S)=H^{2}\left(\mathbb{R}^{3}\right)$. By virtue of $(\mathbf{v})$, we have the orthogonal decomposition

$$
L^{2}=L^{2}\left(\mathbb{R}^{3}\right)=L^{+}+L^{-}
$$

such that $S$ is negative (resp. positive) in $L^{-}$(resp. in $L^{+}$). Let $X=D\left(|S|^{1 / 2}\right)$ be equipped with the inner product

$$
(u, v)=\left(|S|^{1 / 2} u,|S|^{1 / 2} v\right)_{L^{2}}
$$

and norm $\|u\|=\left\||S|^{1 / 2} u\right\|_{L^{2}}$, where $(\cdot, \cdot)_{L^{2}}$ denotes the inner product of $L^{2}$. From (v),

$$
X=H^{1}\left(\mathbb{R}^{3}\right)
$$

with equivalent norms. Therefore, $X$ continuously embeds in $L^{q}\left(\mathbb{R}^{3}\right)$ for all $2 \leq q \leq 6$. In addition, we have the decomposition

$$
X=X^{+}+X^{-},
$$

where $X^{ \pm}=X \cap L^{ \pm}$is orthogonal with respect to both $(\cdot, \cdot)_{L^{2}}$ and $(\cdot, \cdot)$. Therefore, for every $u \in X$, there is a unique decomposition

$$
u=u^{+}+u^{-}, \quad u^{ \pm} \in X^{ \pm}
$$

with $\left(u^{+}, u^{-}\right)=0$ and

$$
\int_{\mathbb{R}^{3}}|\nabla u|^{2} d x+\int_{\mathbb{R}^{3}} V(x) u^{2} d x=\left\|u^{+}\right\|^{2}-\left\|u^{-}\right\|^{2}, \quad u \in X .
$$

For $u \in X$, it is well known (see, e.g., Theorem 2.2.1 of [26]) that the Poisson equation

$$
-\Delta \phi=K_{2}(x) u^{2}
$$

has a unique solution

$$
\phi_{u}(x)=\frac{1}{4 \pi} \int_{\mathbb{R}^{3}} \frac{K_{2}(y) u^{2}(y)}{|x-y|} d y,
$$

$$
\begin{aligned}
& \begin{aligned}
\text { and } \phi_{u} \in & \mathscr{D}^{1,2}\left(\mathbb{R}^{3}\right)=\left\{\left.u \in L^{6}\left(\mathbb{R}^{3}\right)\left|\int_{\mathbb{R}^{3}}\right| \nabla u\right|^{2} d x<\infty\right\} . \\
\qquad(u)= & \frac{1}{2}\left\|u^{+}\right\|^{2}-\frac{1}{2}\left\|u^{-}\right\|^{2} \\
& +\frac{1}{16 \pi} \iint_{\mathbb{R}^{3}} \frac{K_{1}(x) K_{2}(y) u^{2}(x) u^{2}(y)}{|x-y|} d x d y \\
& -\int_{\mathbb{R}^{3}} Q(x) F(u) d x, \quad u \in X .
\end{aligned}
\end{aligned}
$$


Under the assumptions $\left(\mathbf{f}_{\mathbf{1}}\right)$ and $(\mathbf{k}) ; \Phi$ is a $C^{1}$ functional in $X$. The derivative of $\Phi$ is given by

$$
\begin{aligned}
& \left\langle\Phi^{\prime}(u), v\right\rangle \\
& =\left(u^{+}, v\right)-\left(u^{-}, v\right) \\
& \quad+\frac{1}{8 \pi} \iint_{\mathbb{R}^{3}} \frac{K_{1}(x) K_{2}(y) u^{2}(x) u(y) v(y)}{|x-y|} d x d y \\
& \quad+\frac{1}{8 \pi} \iint_{\mathbb{R}^{3}} \frac{K_{1}(x) K_{2}(y) u(x) v(x) u^{2}(y)}{|x-y|} d x d y \\
& \quad-\int_{\mathbb{R}^{3}} Q(x) f(u) v d x, \quad \forall u, v \in X .
\end{aligned}
$$

It is easy to see that if $u$ is a critical point of $\Phi$, then $\left(u, \phi_{u}\right)$ is a solution of (1).

Our functional $\Phi$ does not satisfy the geometric assumptions of the generalized linking theorem (see, e.g., [23, Chapter 6]) because of the term

$$
\frac{1}{16 \pi} \iint_{\mathbb{R}^{3}} \frac{K_{1}(x) K_{2}(y) u^{2}(x) u^{2}(y)}{|x-y|} d x d y .
$$

To overcome this difficulty, we apply the local linking theorem to find critical points of $\Phi$.

Recall that by definition (see [25]), a functional $\Psi$ defined in $X$ has a local linking at 0 with respect to the direct sum decomposition $X=X^{-} \oplus X^{+}$, if there is $\rho>0$ such that

$$
\begin{aligned}
& \Psi(u) \leq 0, \quad \text { for } u \in X^{-},\|u\| \leq \rho, \\
& \Psi(u) \geq 0, \quad \text { for } u \in X^{+},\|u\| \leq \rho .
\end{aligned}
$$

Let $\left\{e_{k}^{ \pm}\right\}$be the total orthonormal sequences in $X^{ \pm}$. We consider two sequences of finite dimensional subspaces

$$
X_{1}^{ \pm} \subset \cdots \subset X_{n}^{ \pm} \subset \cdots \subset X^{ \pm}
$$

where $X_{n}^{ \pm}=\operatorname{span}\left\{e_{1}^{ \pm}, \ldots, e_{n}^{ \pm}\right\}$. It is easy to see that

$$
X^{ \pm}=\overline{\bigcup_{n \in \mathbb{N}} X_{n}^{ \pm}}
$$

For $n \in \mathbb{N}$, let $X_{n}=X_{n}^{-} \oplus X_{n}^{+}$and $\Psi_{n}$ denote the restriction of $\Psi$ on $X_{n}$.

Definition 4. We say that $\Psi \in C^{1}(X)$ satisfies $(C)^{*}$ condition if any sequence $\left\{u_{n}\right\} \subset X$ such that

$$
\begin{gathered}
u_{n} \in X_{n}, \\
\sup _{n} \Psi\left(u_{n}\right)<\infty, \\
\left(1+\left\|u_{n}\right\|\right)\left\|\Psi_{n}^{\prime}\left(u_{n}\right)\right\|_{X_{n}^{*}} \longrightarrow 0
\end{gathered}
$$

contains a subsequence that converges to a critical point of $\Psi$.

From [27, Theorem 2.2], we have the following.
Theorem 5. Suppose that $\Psi \in C^{1}(X)$ has a local linking at 0 , $\Phi$ satisfies $(C)^{*}$ condition, $\Psi$ maps bounded sets into bounded sets, and for every $m \in \mathbb{N}$,

$$
\Psi(u) \longrightarrow-\infty, \quad \text { as }\|u\| \longrightarrow \infty, u \in X^{-} \oplus X_{m}^{+} .
$$

Then, $\Psi$ has a nontrivial critical point.

Lemma 6. The functional $\Phi$ has a local linking in 0 with respect to the direct sum decomposition $X=X^{+} \oplus X^{-}$.

Proof. From the Hardy-littlewood-Sobolev inequality (see, e.g., [28]), we infer that there exists $C>0$ such that for every $u \in X$,

$$
\begin{aligned}
& \iint_{\mathbb{R}^{3}} \frac{K_{1}(x) K_{2}(y) u^{2}(x) u^{2}(y)}{|x-y|} d x d y \\
& \quad \leq C\left\|K_{1} u^{2}\right\|_{L^{6 / 5}\left(\mathbb{R}^{3}\right)}\left\|K_{2} u^{2}\right\|_{L^{6 / 5}\left(\mathbb{R}^{3}\right)} \\
& \quad=C\left\|K_{1}^{1 / 2} u\right\|_{L^{12 / 5}\left(\mathbb{R}^{3}\right)}^{2}\left\|K_{2}^{1 / 2} u\right\|_{L^{12 / 5}\left(\mathbb{R}^{3}\right)^{2}}^{2}
\end{aligned}
$$

From $(\mathbf{k})$, we deduce that $K_{i}$ is bounded in $\mathbb{R}^{3}, i=1,2$. Therefore, by the Sobolev inequality, we have

$$
\left\|K_{i}^{1 / 2} u\right\|_{L^{12 / 5}\left(\mathbb{R}^{3}\right)} \leq C\|u\|_{L^{12 / 5}\left(\mathbb{R}^{3}\right)} \leq C\|u\|, \quad i=1,2 .
$$

It follows that

$$
\iint_{\mathbb{R}^{3}} \frac{K_{1}(x) K_{2}(y) u^{2}(x) u^{2}(y)}{|x-y|} d x d y \leq C\|u\|^{4}
$$

for some $C>0$.

From $\left(\mathbf{f}_{1}\right)$ and $\left(\mathbf{f}_{2}\right)$, we deduce that for any $\epsilon>0$, there exists $C_{\epsilon}>0$ such that

$$
|F(t)| \leq \epsilon t^{2}+C_{\epsilon}|t|^{p}, \quad \forall t \in \mathbb{R} .
$$

This together with the fact that $Q$ is a bounded function in $\mathbb{R}^{3}$ (see (k)) implies that there exists $C^{\prime}>0$ such that

$$
\left|\int_{\mathbb{R}^{3}} Q(x) F(u) d x\right| \leq C^{\prime} \epsilon\|u\|^{2}+C^{\prime} C_{\epsilon}\|u\|^{p} .
$$

Combining (28), (30), and the definition of $\Phi$ (see (18)), we get that for any $u \in X^{+}$,

$$
\begin{aligned}
\Phi(u) & \geq \frac{1}{2}\|u\|^{2}-C^{\prime} \epsilon\|u\|^{2}-C^{\prime} C_{\epsilon}\|u\|^{p} \\
& =\left(\frac{1}{2}-C^{\prime} \epsilon\right)\|u\|^{2}-C^{\prime} C_{\epsilon}\|u\|^{p}
\end{aligned}
$$

and for any $u \in X^{-}$,

$$
\begin{aligned}
\Phi(u) & \leq-\frac{1}{2}\|u\|^{2}+\frac{C}{16 \pi}\|u\|^{4}-C^{\prime} \epsilon\|u\|^{2}-C^{\prime} C_{\epsilon}\|u\|^{p} \\
& =-\left(\frac{1}{2}-C^{\prime} \varepsilon\right)\|u\|^{2}+\frac{C}{16 \pi}\|u\|^{4}+C^{\prime} C_{\epsilon}\|u\|^{p} .
\end{aligned}
$$

Choose $\epsilon=1 / 4 C^{\prime}$. Then, from the above two inequalities, we deduce that we can choose small $\rho>0$ such that $\Phi$ satisfies (21). Therefore, $\Phi$ has a local linking in 0 with respect to the direct sum decomposition $X=X^{+} \oplus X^{-}$. 
Lemma 7. Under the assumptions $(\mathbf{v}),(\mathbf{k})$, and $\left(\mathbf{f}_{\mathbf{1}}\right)-\left(\mathbf{f}_{\mathbf{3}}\right)$, the functional $\Phi$ satisfies the $(C)^{*}$ condition.

Proof. Let $\left\{u_{n}\right\}$ be a $(C)_{c}^{*}$ sequence; that is, $\sup _{n} \Phi\left(u_{n}\right) \leq c$ and $\left(1+\left\|u_{n}\right\|\right)\left\|\Phi^{\prime}\left(u_{n}\right)\right\|_{X_{n}^{*}} \rightarrow 0$. have

First, we prove that $\left\{u_{n}\right\}$ is bounded in $X$. From (7), we

$$
\begin{aligned}
o(1)+2 c \geq & 2 \Phi\left(u_{n}\right)-\left\langle\Phi^{\prime}\left(u_{n}\right), u_{n}\right\rangle \\
= & -\frac{1}{8 \pi} \iint_{\mathbb{R}^{3}} \frac{K_{1}(x) K_{2}(y) u_{n}^{2}(x) u_{n}^{2}(y)}{|x-y|} d x d y \\
& +\int_{\mathbb{R}^{3}} Q(x)\left(u_{n} f\left(u_{n}\right)-2 F\left(u_{n}\right)\right) d x \\
\geq & -\frac{1}{8 \pi} \iint_{\mathbb{R}^{3}} \frac{K_{1}(x) K_{2}(y) u_{n}^{2}(x) u_{n}^{2}(y)}{|x-y|} d x d y \\
& +A \int_{\mathbb{R}^{3}} Q(x)\left|u_{n}\right|^{p} d x .
\end{aligned}
$$

From (26), we have

$$
\begin{aligned}
& \iint_{\mathbb{R}^{3}} \frac{K_{1}(x) K_{2}(y) u_{n}^{2}(x) u_{n}^{2}(y)}{|x-y|} d x d y \\
& \quad \leq C\left\|K_{1} u^{2}\right\|_{L^{6 / 5}\left(\mathbb{R}^{3}\right)}\left\|K_{2} u^{2}\right\|_{L^{6 / 5}\left(\mathbb{R}^{3}\right)^{\circ}}
\end{aligned}
$$

By the Hölder inequality and (4), we have

$$
\begin{aligned}
\int_{\mathbb{R}^{3}} & K_{i}^{6 / 5}(x)\left|u_{n}(x)\right|^{12 / 5} d x \\
\leq & \left(\int_{\mathbb{R}^{3}} Q(x)\left|u_{n}(x)\right|^{p} d x\right)^{12 / 5 p} \\
& \times\left(\int_{\mathbb{R}^{3}} K_{i}^{6 p /(5 p-12)}(x) Q^{-12 /(5 p-12)}(x) d x\right)^{1-(12 / 5 p)} \\
\leq & C\left(\int_{\mathbb{R}^{3}} Q(x)\left|u_{n}(x)\right|^{p} d x\right)^{12 / 5 p}, \quad i=1,2 .
\end{aligned}
$$

Combining (34) and (35), we get that

$$
\begin{gathered}
\iint_{\mathbb{R}^{3}} \frac{K_{1}(x) K_{2}(y) u_{n}^{2}(x) u_{n}^{2}(y)}{|x-y|} d x d y \\
\quad \leq C\left(\int_{\mathbb{R}^{3}} Q(x)\left|u_{n}(x)\right|^{p} d x\right)^{4 / p} .
\end{gathered}
$$

This together with (33) yields that $\int_{\mathbb{R}^{3}} Q(x)\left|u_{n}(x)\right|^{p} d x$ is bounded.
Second, we prove that $\left\|u_{n}\right\|$ is bounded. We have

$$
\begin{aligned}
o(1)=\left\langle\Phi^{\prime}\left(u_{n}\right), u_{n}^{+}-\right. & \left.u_{n}^{-}\right\rangle \\
=\left\|u_{n}\right\|^{2}-\int_{\mathbb{R}^{3}} Q & (x) f\left(u_{n}\right)\left(u_{n}^{+}-u_{n}^{-}\right) d x \\
+\frac{1}{8 \pi} \iint_{\mathbb{R}^{3}}( & \left(K_{1}(x) K_{2}(y) u_{n}^{2}(x) u_{n}(y)\right. \\
& \left.\times\left(u_{n}^{+}(y)-u_{n}^{-}(y)\right)\right) \\
& \left.\times(|x-y|)^{-1}\right) d x d y \\
+\frac{1}{8 \pi} \iint_{\mathbb{R}^{3}}( & K_{1}(x) K_{2}(y) u_{n}(x) \\
& \left.\times\left(u_{n}^{+}(x)-u_{n}^{-}(x)\right) u_{n}^{2}(y)\right) \\
& \left.\times(|x-y|)^{-1}\right) d x d y .
\end{aligned}
$$

From (5) and the Hölder inequality, we get that for any $R>0$,

$$
\begin{aligned}
& \int_{\mathbb{R}^{3}} Q(x) f\left(u_{n}\right)\left(u_{n}^{+}-u_{n}^{-}\right) d x \\
& \leq C \int_{\mathbb{R}^{3}} Q(x)\left|u_{n}\right| \cdot\left|u_{n}^{+}-u_{n}^{-}\right| d x \\
& \quad+C \int_{\mathbb{R}^{3}} Q(x)\left|u_{n}\right|^{p-1}\left|u_{n}^{+}-u_{n}^{-}\right| d x \\
& \leq C \int_{\left\{x \in \mathbb{R}^{3}|| x \mid>R\right\}} Q(x)\left|u_{n}\right| \cdot\left|u_{n}^{+}-u_{n}^{-}\right| d x \\
& \quad+C \int_{\left\{x \in \mathbb{R}^{3}|| x \mid \leq R\right\}} Q(x)\left|u_{n}\right| \cdot\left|u_{n}^{+}-u_{n}^{-}\right| d x \\
& \quad+C\left(\int_{\mathbb{R}^{3}} Q(x)\left|u_{n}\right|^{p} d x\right)^{(p-1) / p} \\
& \quad \times\left(\int_{\mathbb{R}^{3}} Q(x)\left|u_{n}^{+}-u_{n}^{-}\right|^{p} d x\right)^{1 / p} .
\end{aligned}
$$

Since $\int_{\mathbb{R}^{3}} Q(x)\left|u_{n}\right|^{p} d x$ is bounded,

$$
\begin{aligned}
& C\left(\int_{\mathbb{R}^{3}} Q(x)\left|u_{n}\right|^{p} d x\right)^{(p-1) / p}\left(\int_{\mathbb{R}^{3}} Q(x)\left|u_{n}^{+}-u_{n}^{-}\right|^{p} d x\right)^{1 / p} \\
& \quad \leq C\left\|u_{n}\right\| .
\end{aligned}
$$

From $\lim _{|x| \rightarrow \infty} Q(x)=0$ (see $\left.(\mathbf{k})\right)$ and the Sobolev inequality, we get that there exists $R>0$ such that

$$
\begin{aligned}
& C \int_{\left\{x \in \mathbb{R}^{3}|| x \mid>R\right\}} Q(x)\left|u_{n}\right| \cdot\left|u_{n}^{+}-u_{n}^{-}\right| d x \\
& \quad \leq C \sup _{|x|>R} Q \cdot \int_{\left\{x \in \mathbb{R}^{3}|| x \mid>R\right\}}\left|u_{n}\right| \cdot\left|u_{n}^{+}-u_{n}^{-}\right| d x \\
& \quad \leq \quad \sup _{|x|>R} Q \cdot\left\|u_{n}\right\|^{2} \leq \frac{1}{2}\left\|u_{n}\right\|^{2} .
\end{aligned}
$$


From the Hölder and Sobolev inequalities and boundedness of $\int_{\mathbb{R}^{3}} Q(x)\left|u_{n}\right|^{p} d x$, we have

$$
\begin{aligned}
& C \int_{\left\{x \in \mathbb{R}^{3}|| x \mid \leq R\right\}} Q(x)\left|u_{n}\right| \cdot\left|u_{n}^{+}-u_{n}^{-}\right| d x \\
& \leq C\left(\int_{\left\{x \in \mathbb{R}^{3}|| x \mid \leq R\right\}} Q(x) d x\right)^{p /(p-2)}\left(\int_{\mathbb{R}^{3}} Q(x)\left|u_{n}\right|^{p} d x\right)^{1 / p} \\
& \quad \times\left(\int_{\mathbb{R}^{3}} Q(x)\left|u_{n}^{+}-u_{n}^{-}\right|^{p} d x\right)^{1 / p} \leq D_{R}\left\|u_{n}\right\|,
\end{aligned}
$$

where $D_{R}$ is a positive constant that depends only on $R$. Combining (38)-(41), we infer that there exists $R>0$ such that

$$
\int_{\mathbb{R}^{3}} Q(x) f\left(u_{n}\right)\left(u_{n}^{+}-u_{n}^{-}\right) d x \leq \frac{1}{2}\left\|u_{n}\right\|^{2}+\left(D_{R}+C\right)\left\|u_{n}\right\| .
$$

From the Hardy-Littlewood-Sobolev and Hölder inequalities, we get that

$$
\begin{aligned}
& \iint_{\mathbb{R}^{3}} \frac{K_{1}(x) K_{2}(y) u_{n}^{2}(x) u_{n}(y)\left(u_{n}^{+}(y)-u_{n}^{-}(y)\right)}{|x-y|} d x d y \\
& \quad \leq C\left\|K_{1} u_{n}^{2}\right\|_{L^{6 / 5}\left(\mathbb{R}^{3}\right)}\left\|K_{2} u_{n}\left(u_{n}^{+}-u_{n}^{-}\right)\right\|_{L^{6 / 5}\left(\mathbb{R}^{3}\right)} \\
& \quad \leq C\left\|K_{1}^{1 / 2} u_{n}\right\|_{L^{12 / 5}\left(\mathbb{R}^{3}\right)}^{2}\left\|K_{2}^{1 / 2} u_{n}\right\|_{L^{12 / 5}\left(\mathbb{R}^{3}\right)} \\
& \quad \times\left\|K_{2}^{1 / 2}\left(u_{n}^{+}-u_{n}^{-}\right)\right\|_{L^{12 / 5}\left(\mathbb{R}^{3}\right)^{*}}
\end{aligned}
$$

From (43), (35), and the boundedness of $\int_{\mathbb{R}^{3}} Q(x)\left|u_{n}\right|^{p} d x$, we get that there exists a constant $C>0$ such that

$$
\begin{aligned}
& \iint_{\mathbb{R}^{3}} \frac{K_{1}(x) K_{2}(y) u_{n}^{2}(x) u_{n}(y)\left(u_{n}^{+}(y)-u_{n}^{-}(y)\right)}{|x-y|} d x d y \\
& \quad \leq C\left\|K_{2}^{1 / 2}\left(u_{n}^{+}-u_{n}^{-}\right)\right\|_{L^{12 / 5}\left(\mathbb{R}^{3}\right)} \leq C\left\|u_{n}\right\| .
\end{aligned}
$$

Similarly, we have

$$
\begin{aligned}
& \iint_{\mathbb{R}^{3}} \frac{K_{1}(x) K_{2}(y) u_{n}(x)\left(u_{n}^{+}(x)-u_{n}^{-}(x)\right) u_{n}^{2}(y)}{|x-y|} d x d y \\
& \quad \leq C\left\|u_{n}\right\| .
\end{aligned}
$$

Combining (37), (42), (44), and (45), we infer that $\left\|u_{n}\right\|$ is bounded.

Finally, we prove that $\left\{u_{n}\right\}$ has a convergent subsequence. Up to a subsequence, we assume that $u_{n} \rightarrow u$ in $X$. Then, $u$ is a critical point of $\Phi$. From $\left(\mathbf{f}_{1}\right)$,

$$
\int_{\mathbb{R}^{3}} Q(x) f\left(u_{n}\right) u^{+} d x \longrightarrow \int_{\mathbb{R}^{3}} Q(x) f(u) u^{+} d x
$$

and from $\lim _{|x| \rightarrow \infty} Q(x)=0$ and $u_{n} \rightarrow u$,

$$
\begin{aligned}
& \left|\int_{\mathbb{R}^{3}} Q(x) f\left(u_{n}\right)\left(u_{n}^{+}-u^{+}\right) d x\right| \\
& \quad \leq C \int_{\mathbb{R}^{3}} Q(x)\left|u_{n}\right|^{p-1}\left|u_{n}^{+}-u^{+}\right| d x \longrightarrow 0 .
\end{aligned}
$$

Therefore,

$$
\int_{\mathbb{R}^{3}} Q(x) f\left(u_{n}\right) u_{n}^{+} d x-\int_{\mathbb{R}^{3}} Q(x) f(u) u^{+} d x \longrightarrow 0 .
$$

By $u_{n} \rightarrow u$ and $\lim _{|x| \rightarrow \infty} K_{i}(x)=0, i=1,2$, we get that

$$
\begin{aligned}
& \mid \iint_{\mathbb{R}^{3}} \frac{K_{1}(x) K_{2}(y) u_{n}^{2}(x) u_{n}(y) u_{n}^{+}(y)}{|x-y|} d x d y \\
& \quad-\iint_{\mathbb{R}^{3}} \frac{K_{1}(x) K_{2}(y) u^{2}(x) u(y) u^{+}(y)}{|x-y|} d x d y \mid \\
& \leq\left\|K_{1}\left(u_{n}^{2}-u^{2}\right)\right\|_{L^{6 / 5}\left(\mathbb{R}^{3}\right)}\left\|K_{2}\left(u_{n} u_{n}^{+}-u u^{+}\right)\right\|_{L^{6 / 5}\left(\mathbb{R}^{3}\right)} \rightarrow 0 .
\end{aligned}
$$

Similarly,

$$
\begin{aligned}
& \mid \iint_{\mathbb{R}^{3}} \frac{K_{1}(x) K_{2}(y) u_{n}(x) u_{n}^{+}(x) u_{n}^{2}(y)}{|x-y|} d x d y \\
& \quad-\iint_{\mathbb{R}^{3}} \frac{K_{1}(x) K_{2}(y) u(x) u^{+}(x) u^{2}(y)}{|x-y|} d x d y \mid \rightarrow 0 .
\end{aligned}
$$

By (48)-(50), $\left\langle\Phi^{\prime}(u), u^{+}\right\rangle=0$, and

$$
\begin{aligned}
o(1)= & \left\langle\Phi^{\prime}\left(u_{n}\right), u_{n}^{+}\right\rangle \\
= & \left\|u_{n}^{+}\right\|^{2}-\int_{\mathbb{R}^{3}} Q(x) f\left(u_{n}\right) u_{n}^{+} d x \\
& +\frac{1}{8 \pi} \iint_{\mathbb{R}^{3}} \frac{K_{1}(x) K_{2}(y) u_{n}^{2}(x) u_{n}(y) u_{n}^{+}(y)}{|x-y|} d x d y \\
& +\frac{1}{8 \pi} \iint_{\mathbb{R}^{3}} \frac{K_{1}(x) K_{2}(y) u_{n}(x) u_{n}^{+}(x) u_{n}^{2}(y)}{|x-y|} d x d y,
\end{aligned}
$$

we get that $u_{n}^{+} \rightarrow u^{+}$in $X$. The same argument implies that $u_{n}^{-} \rightarrow u^{-}$in $X$. Therefore, $u_{n} \rightarrow u$ in $X$.

Remark 8. From the proof of this theorem, we infer that $\Phi$ also satisfies the Cerami condition; that is, if $\left\{u_{n}\right\} \subset X$ satisfies

$$
\sup _{n} \Phi\left(u_{n}\right)<+\infty, \quad\left(1+\left\|u_{n}\right\|\right)\left\|\Phi^{\prime}\left(u_{n}\right)\right\|_{X^{*}} \longrightarrow 0
$$

then $\left\{u_{n}\right\}$ contains a convergent subsequence.

Lemma 9. The functional $\Phi$ satisfies (25). 
Proof. If the functional does not satisfy (25), then there exist $M>0, m \in \mathbb{N}$, and $u_{n} \in X_{m}^{+} \oplus X^{-}$such that $\left\|u_{n}\right\| \rightarrow \infty$ and

$$
\begin{aligned}
\Phi\left(u_{n}\right)= & \frac{1}{2}\left\|u_{n}^{+}\right\|^{2}-\frac{1}{2}\left\|u_{n}^{-}\right\|^{2} \\
& +\frac{1}{16 \pi} \iint_{\mathbb{R}^{3}} \frac{K_{1}(x) K_{2}(y) u_{n}^{2}(x) u_{n}^{2}(y)}{|x-y|} d x d y \\
& -\int_{\mathbb{R}^{3}} Q(x) F\left(u_{n}\right) d x \geq-M .
\end{aligned}
$$

This together with (36) and (9) yields

$$
\begin{gathered}
\frac{1}{2}\left\|u_{n}^{+}\right\|^{2}-\frac{1}{2}\left\|u_{n}^{-}\right\|^{2}+C\left(\int_{\mathbb{R}^{3}} Q(x)\left|u_{n}(x)\right|^{p} d x\right)^{4 / p} \\
-C_{1} \int_{\mathbb{R}^{3}} Q(x)\left|u_{n}(x)\right|^{p} d x \geq-M .
\end{gathered}
$$

Since $p>4$, there exists $D>0$ such that

$$
\begin{aligned}
D- & \frac{C_{1}}{2} \int_{\mathbb{R}^{3}} Q(x)\left|u_{n}(x)\right|^{p} d x \\
\geq & C\left(\int_{\mathbb{R}^{3}} Q(x)\left|u_{n}(x)\right|^{p} d x\right)^{4 / p} \\
& -C_{1} \int_{\mathbb{R}^{3}} Q(x)\left|u_{n}(x)\right|^{p} d x .
\end{aligned}
$$

Then, by (54),

$$
\frac{1}{2}\left\|u_{n}^{+}\right\|^{2}-\frac{1}{2}\left\|u_{n}^{-}\right\|^{2}-\frac{C_{1}}{2} \int_{\mathbb{R}^{3}} Q(x)\left|u_{n}(x)\right|^{p} d x \geq-M-D .
$$

We use $E$ to denote the closure of $X_{m}^{+} \oplus X^{-}$under the norm $\|u\|_{Q, p}=\left(\int_{\mathbb{R}^{3}} Q(x)|u(x)|^{p} d x\right)^{1 / p}$. Since there exists a continuous projection $P: E \rightarrow X_{m}^{+}$, there exists $C_{3}>0$ such that for every $u \in X_{m}^{+} \oplus X^{-}$,

$$
\left(\int_{\mathbb{R}^{3}} Q(x)|u(x)|^{p} d x\right)^{1 / p} \geq C_{3}\left(\int_{\mathbb{R}^{3}} Q(x)\left|u^{+}(x)\right|^{p} d x\right)^{1 / p} .
$$

Since $\left\|u_{n}\right\| \rightarrow \infty$ and $X_{m}^{+}$is finite dimensional, from (56) and (57), we get that

$$
\begin{aligned}
-M-D \leq & \frac{1}{2}\left\|u_{n}^{+}\right\|^{2}-\frac{1}{2}\left\|u_{n}^{-}\right\|^{2} \\
& -\frac{C_{1} C_{3}^{p}}{2} \int_{\mathbb{R}^{3}} Q(x)\left|u_{n}^{+}(x)\right|^{p} d x \longrightarrow-\infty .
\end{aligned}
$$

This contradiction implies that $\Phi$ satisfies (25).

Proof of Theorem 2. The desired result of Theorem 2 can be derived by combining Theorem 5 and Lemmas 6, 7, and 9 .

\section{Proof of Theorem 3}

Recall that $\left\{e_{k}^{ \pm}\right\}$are the total orthonormal sequences in $X^{ \pm}$. For $k=1,2, \ldots$, let

$$
\begin{gathered}
Y_{k}=X^{-} \oplus X_{k}^{+}=X^{-} \oplus \operatorname{span}\left\{e_{1}^{+}, \ldots, e_{k}^{+}\right\}, \\
Z_{k}=\overline{\operatorname{span}\left\{e_{k}^{+}, e_{k+1}^{+}, \ldots\right\}} .
\end{gathered}
$$

Let $P: X \rightarrow X^{-}, Q: X \rightarrow X^{+}$be the orthogonal projections. As [23], on $X$, we define

$$
|\|u\||=\max \left\{\|Q u\|, \sum_{j=1}^{\infty} \frac{1}{2^{k+1}}\left|\left(P u, e_{k}^{-}\right)\right|\right\} .
$$

The topology generated by $|\|\cdot\||$ will be denoted by $\tau$ and all topological notations related to it will include this symbol.

For the proof of Theorem 3, we use the following improved fountain theorem of Batkam and Colin [24, Theorem 12], which is a generalization of the classical fountain theorem of Bartsch [22] (see also [23]).

Theorem 10. Assume that an even functional $\Psi \in C^{1}(X)$ satisfies the following:

(A) $\Psi$ is $\tau$-upper semicontinuous, and the gradient $\nabla \Psi$ is weakly sequentially continuous.

If there exist $\rho_{k}>r_{k}>0$ such that

(i) $b_{k}=\inf _{u \in Z_{k},\|u\|=r_{k}} \Psi(u) \rightarrow+\infty$, as $k \rightarrow \infty$,

(ii) $a_{k}=\max _{u \in Y_{k},\|u\|=\rho_{k}} \Psi(u) \leq 0$,

then there exist $\left\{c_{k}\right\} \subset \mathbb{R}$ and sequences $\left\{u_{k, n}\right\} \subset X$ such that for every $k \in \mathbb{N}, c_{k} \geq b_{k}$ and

$$
\begin{aligned}
\Psi\left(u_{k, n}\right) \longrightarrow c_{k}, \quad\left(1+\left\|u_{k, n}\right\|\right)\left\|\Psi^{\prime}\left(u_{k, n}\right)\right\|_{X^{*}} & \longrightarrow 0, \\
\text { as } n & \longrightarrow \infty .
\end{aligned}
$$

Remark 11. In [24], the result of this fountain theorem is

$$
\Psi\left(u_{k, n}\right) \longrightarrow c_{k}, \quad\left\|\Psi^{\prime}\left(u_{k, n}\right)\right\|_{X^{*}} \longrightarrow 0, \quad \text { as } n \longrightarrow \infty .
$$

Since the deformation theorem is still valid under the Cerami condition (see, e.g., [27]), replacing the pseudogradient vector field $2\|\nabla \Psi(v)\|^{-2} \nabla \Psi(v)$ in page 442 of [24] by the Cerami-type pseudogradient vector field $(1+\|v\|) \nabla \Psi(v)$, we see that similar to many critical point theorems, the result of the fountain theorem in [24] can be improved as (61).

Proof of Theorem 3. (1) Let us prove that $\Phi$ is $\tau$-upper semicontinuous. Assume that $\left|\left\|u_{n}-u\right\|\right| \rightarrow 0$ and $c \leq \Phi\left(u_{n}\right)$. Since $u_{n}^{+}=Q u_{n} \rightarrow Q u, u_{n}^{-}=P u_{n}$ are bounded so that $P u_{n} \rightarrow P u$, it follows that $u_{n} \rightarrow u$ in $X$. Since $\lim _{|x| \rightarrow \infty} Q(x)=0$, from $\left(\mathbf{f}_{1}\right)$ and $u_{n} \rightarrow u$, we obtain

$$
\int_{\mathbb{R}^{3}} Q(x) F\left(u_{n}\right) d x \rightarrow \int_{\mathbb{R}^{3}} Q(x) F(u) d x .
$$


In addition, from the proof of (49) we infer that

$$
\begin{aligned}
& \mid \iint_{\mathbb{R}^{3}} \frac{K_{1}(x) K_{2}(y) u_{n}^{2}(x) u_{n}^{2}(y)}{|x-y|} d x d y \\
& \quad-\iint_{\mathbb{R}^{3}} \frac{K_{1}(x) K_{2}(y) u^{2}(x) u^{2}(y)}{|x-y|} d x d y \mid \rightarrow 0 .
\end{aligned}
$$

Using the Fatou lemma, we obtain

$$
\begin{aligned}
& -\Phi(u) \\
& =\frac{\|P u\|^{2}}{2}-\frac{\|Q u\|^{2}}{2}-\frac{1}{16 \pi} \\
& \quad \times \iint_{\mathbb{R}^{3}} \frac{K_{1}(x) K_{2}(y) u^{2}(x) u^{2}(y)}{|x-y|} d x d y \\
& +\int_{\mathbb{R}^{3}} Q(x) F(u) d x \\
& \leq \liminf _{n \rightarrow \infty} \frac{\left\|P u_{n}\right\|^{2}}{2}-\frac{\left\|Q u_{n}\right\|^{2}}{2} \\
& +\liminf _{n \rightarrow \infty}-\frac{1}{16 \pi} \iint_{\mathbb{R}^{3}} \frac{K_{1}(x) K_{2}(y) u_{n}^{2}(x) u_{n}^{2}(y)}{|x-y|} d x d y \\
& +\int_{\mathbb{R}^{3}} Q-c .
\end{aligned}
$$

(2) The proof that $\nabla \Psi$ is weakly sequentially continuous is similar to that in the proof of Lemma 6.15 of [23].

(3) Verification of (i) for $\Phi$ : since $\lim _{|x| \rightarrow \infty} Q(x)=0$, we infer that if $u_{k} \rightarrow 0$ in $X$, then

$$
\int_{\mathbb{R}^{3}} Q(x)\left|u_{n}\right|^{p} d x \longrightarrow 0 .
$$

It follows that

$$
\beta_{k}=\sup _{u \in Z_{k},\|u\|=1}\|u\|_{Q, p} \longrightarrow 0, \quad \text { as } \quad k \longrightarrow \infty,
$$

where

$$
\|u\|_{Q, q}=\left(\int_{\mathbb{R}^{3}} Q(x)|u|^{q} d x\right)^{1 / q}
$$

From $\left(\mathbf{f}_{1}\right)$ and $\left(\mathbf{f}_{2}\right)$, we deduce that for any $\epsilon>0$, there exists $C_{\epsilon}>0$ such that

$$
|F(t)| \leq \epsilon|t|^{2}+C_{\epsilon}|t|^{p}, \quad \forall t \in \mathbb{R} .
$$

For $u \in Z_{k}$, by the Sobolev inequality and (67),

$$
\begin{aligned}
\Phi(u)= & \frac{1}{2}\|u\|^{2} \\
& +\frac{1}{16 \pi} \iint_{\mathbb{R}^{3}} \frac{K_{1}(x) K_{2}(y) u^{2}(x) u^{2}(y)}{|x-y|} d x d y \\
& -\int_{\mathbb{R}^{3}} Q(x) F(u) d x \\
\geq & \frac{1}{2}\|u\|^{2}-\epsilon\|u\|_{\mathrm{Q}, 2}^{2}-C_{\epsilon}\|u\|_{Q, p}^{p} \\
\geq & \frac{1}{2}\|u\|^{2}-C \epsilon\|u\|^{2}-C_{\epsilon} \beta_{k}^{p}\|u\|^{p} .
\end{aligned}
$$

Choosing $\epsilon=1 / 4 C$ and letting $r_{k}=\left(2 C_{\epsilon} p \beta_{k}^{p}\right)^{1 /(2-p)}$, we get from (70) that for $\|u\|=r_{k}$,

$$
\Phi(u) \geq \frac{1}{2}\left(\frac{1}{2}-\frac{1}{p}\right)\left(2 C_{\epsilon} p \beta_{k}^{p}\right)^{2 /(2-p)} .
$$

Since $\beta_{k} \rightarrow 0$ and $p>2$, it follows that

$$
b_{k}=\inf _{u \in Z_{k},\|u\|=r_{k}} \Phi(u) \longrightarrow+\infty .
$$

(4) Verification of (ii) for $\Phi$ : since $Y_{k}=X^{-} \oplus X_{k}^{+}$, (ii) is a direct consequence of Lemma 9.

Finally, from $\left(\mathbf{f}_{4}\right)$, we obtain that $\Phi$ is an even functional. Then, from (1)-(4) and Remark 8, we deduce that for every $k$, there exists a critical point $u_{k}$ of $\Phi$ such that $\Phi\left(u_{k}\right) \geq b_{k}$. Therefore, (1) has infinitely many solutions $\left\{\left(u_{k}, \phi_{u_{k}}\right)\right\}$.

\section{Conflict of Interests}

The authors declare that there is no conflict of interests regarding the publication of this paper.

\section{Acknowledgments}

The authors would like to thank the anonymous referees for their comments and suggestions on the paper. Shaowei Chen was supported by Science Foundation of Huaqiao University and Promotion Program for Young and Middle-aged Teacher in Science and Technology Research of Huaqiao University.

\section{References}

[1] B. Simon, "Schrödinger semigroups," Bulletin of the American Mathematical Society, vol. 7, pp. 447-526, 1982.

[2] T. D'Aprile and D. Mugnai, "Solitary waves for nonlinear Klein-Gordon-Maxwell and Schrödinger-Maxwell equations," Proceedings of the Royal Society of Edinburgh, vol. 134, no. 5, pp. 893-906, 2004.

[3] Ó. Sánchez and J. Soler, "Long-time dynamics of the Schrödinger-Poisson-Slater system," Journal of Statistical Physics, vol. 114, no. 1-2, pp. 179-204, 2004.

[4] C. O. Alves, M. A. S. Souto, and S. H. M. Soares, "SchrödingerPoisson equations without Ambrosetti-Rabinowitz condition," Journal of Mathematical Analysis and Applications, vol. 377, no. 2, pp. 584-592, 2011. 
[5] A. Azzollini and A. Pomponio, "Ground state solutions for the nonlinear Schrödinger-Maxwell equations," Journal of Mathematical Analysis and Applications, vol. 345, no. 1, pp. 90-108, 2008.

[6] V. Benci and D. Fortunato, "An eigenvalue problem for the Schrödinger-Maxwell equations," Topological Methods in Nonlinear Analysis, vol. 11, pp. 283-293, 1998.

[7] V. Benci, D. Fortunato, A. Masiello, and L. Pisani, "Solitons and the electromagnetic field," Mathematische Zeitschrift, vol. 232, no. 1, pp. 73-102, 1999.

[8] S.-J. Chen and C.-L. Tang, "High energy solutions for the superlinear Schrödinger-Maxwell equations," Nonlinear Analysis, Theory, Methods \& Applications, vol. 71, no. 10, pp. 49274934, 2009.

[9] G. M. Coclite, "A multiplicity result for the nonlinear Schrödinger-Maxwell equations," Communications on Applied Analysis, vol. 7, pp. 417-423, 2003.

[10] Y. Jiang and H.-S. Zhou, "Schrödinger-Poisson system with steep potential well," Journal of Differential Equations, vol. 251, no. 3, pp. 582-608, 2011.

[11] Q. Li, H. Su, and Z. Wei, "Existence of infinitely many large solutions for the nonlinear Schrödinger-Maxwell equations," Nonlinear Analysis, Theory, Methods \& Applications, vol. 72, no. 11, pp. 4264-4270, 2010.

[12] H. Kikuchi, "On the existence of a solution for elliptic system related to the Maxwell-Schrödinger equations," Nonlinear Analysis, Theory, Methods \& Applications, vol. 67, no. 5, pp. 14451456, 2007.

[13] J. Sun, H. Chen, and J. J. Nieto, "On ground state solutions for some non-autonomous Schrödinger-Poisson systems," Journal of Differential Equations, vol. 252, no. 5, pp. 3365-3380, 2012.

[14] Z. Wang and H.-S. Zhou, "Positive solution for a nonlinear stationary Schrödinger-poisson system in $\mathbb{R} 3$," Discrete and Continuous Dynamical Systems, vol. 18, no. 4, pp. 809-816, 2007.

[15] M.-H. Yang and Z.-Q. Han, "Existence and multiplicity results for the nonlinear SchrödingerPoisson systems," Nonlinear Analysis: Real World Applications, vol. 13, no. 3, pp. 1093-1101, 2012.

[16] J. Zhang, "On the Schrödinger-Poisson equations with a general nonlinearity in the critical growth," Nonlinear Analysis: Theory, Methods \& Applications, vol. 75, pp. 6391-6401, 2012.

[17] A. Ambrosetti and D. Ruiz, "Multiple bound states for the Schrödinger-Poisson problem," Communications in Contemporary Mathematics, vol. 10, no. 3, pp. 391-404, 2008.

[18] T. D’Aprile and D. Mugnai, "Non-existence results for the coupled Klein-Gordon-Maxwell equations," Advanced Nonlinear Studies, vol. 4, no. 3, pp. 307-322, 2004.

[19] D. Ruiz, "The Schrödinger-Poisson equation under the effect of a nonlinear local term," Journal of Functional Analysis, vol. 237, no. 2, pp. 655-674, 2006.

[20] L. Zhao and F. Zhao, "On the existence of solutions for the Schrödinger-Poisson equations," Journal of Mathematical Analysis and Applications, vol. 346, pp. 155-169, 2008.

[21] H. Chen and S. Liu, "Standing waves with large frequency for 4-superlinear Schrödinger-Poisson systems," Annali di Matematica Pura ed Applicata, 2013.

[22] T. Bartsch, "Infinitely many solutions of a symmetric Dirichlet problem," Nonlinear Analysis: Theory, Methods \& Applications, vol. 20, no. 10, pp. 1205-1216, 1993.

[23] M. Willem, Minimax Theorems, Progress in Nonlinear Differential Equations and Their Applications, vol. 24, Birkhäuser, Boston, Mass, USA, 1996.
[24] C. J. Batkama and F. Colin, "Generalized Fountain theorem and applications to strongly indefinite semilinear problems," Journal of Mathematical Analysis and Applications, vol. 405, pp. 438452, 2013.

[25] S. J. Li and M. Willem, "Applications of local linking to critical point theory," Journal of Mathematical Analysis and Applications, vol. 189, no. 1, pp. 6-32, 1995.

[26] L. C. Evans, Partial Differential Equations, vol. 19 of Graduate Studies in Mathematics, American Mathematical Society, Providence, RI, USA, 1998.

[27] S. Luan and A. Mao, "Periodic solutions for a class of nonautonomous Hamiltonian systems," Nonlinear Analysis, Theory, Methods \& Applications, vol. 61, no. 8, pp. 1413-1426, 2005.

[28] E. lieb, "Sharp constants in the Hrady-Littlewood-Sobolev and related inequalities," Annals of Mathematics, vol. 118, pp. 449$374,1983$. 


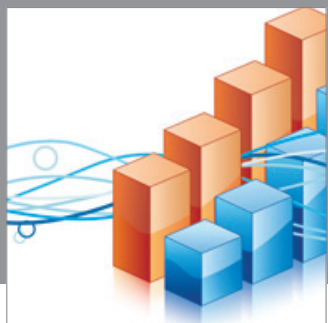

Advances in

Operations Research

mansans

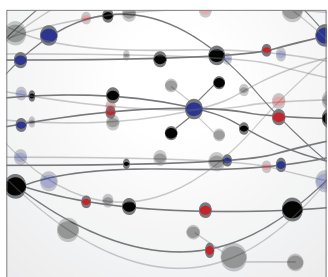

The Scientific World Journal
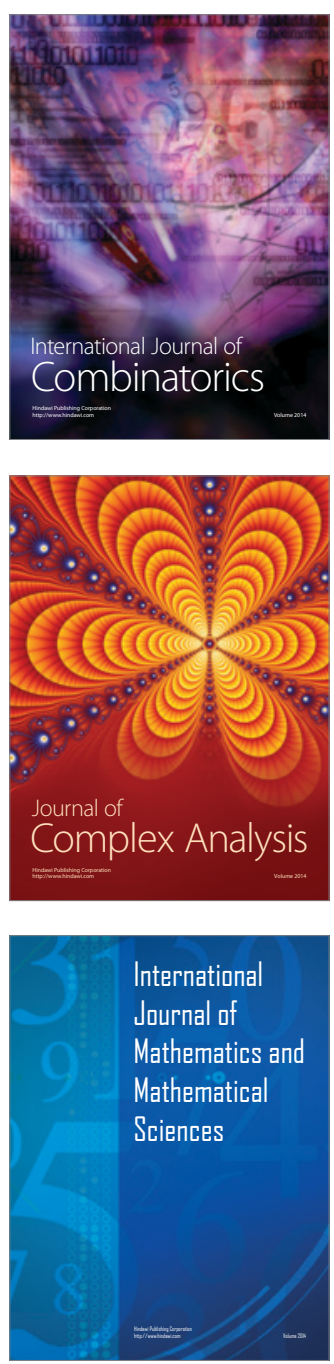
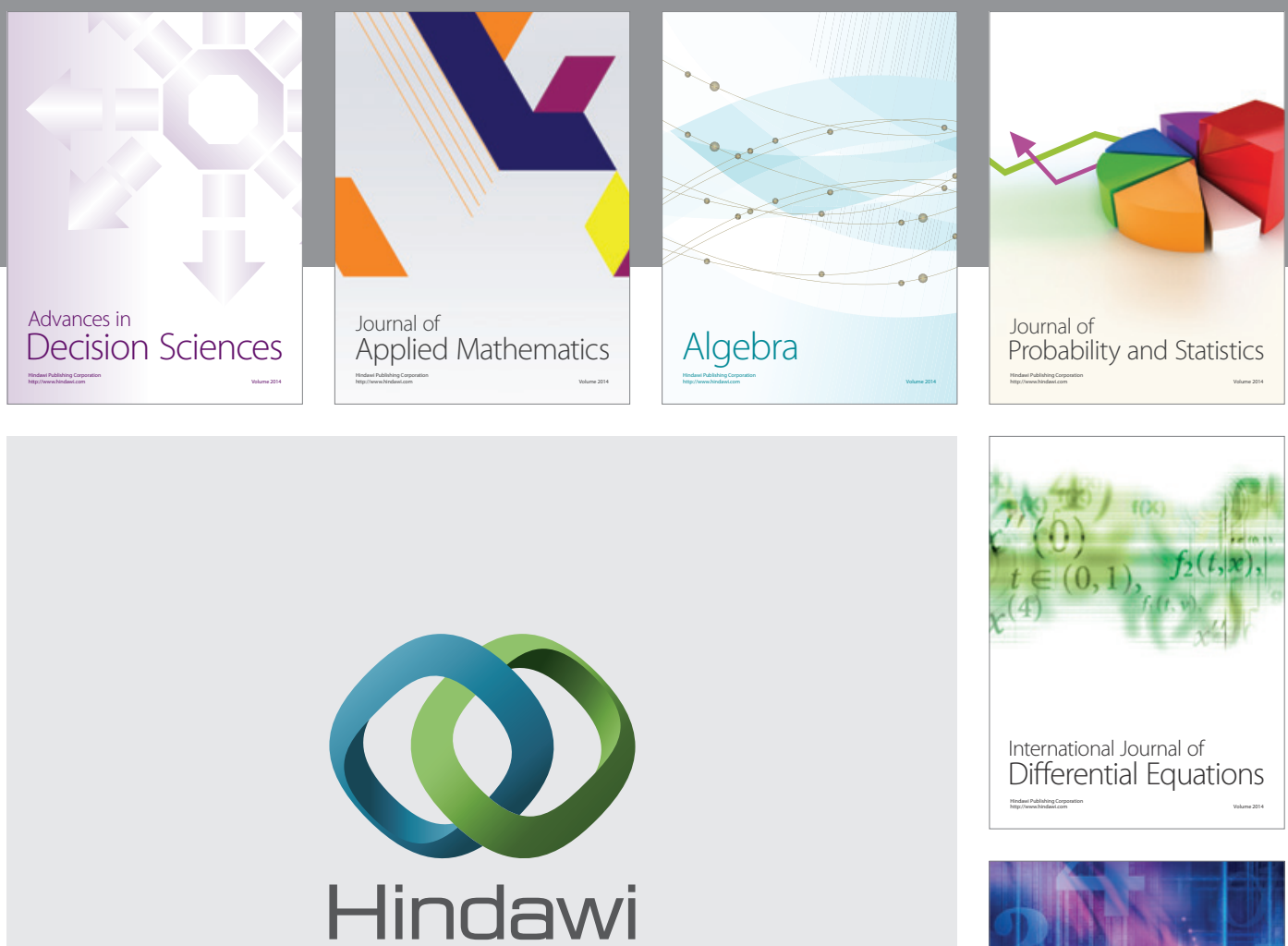

Submit your manuscripts at http://www.hindawi.com
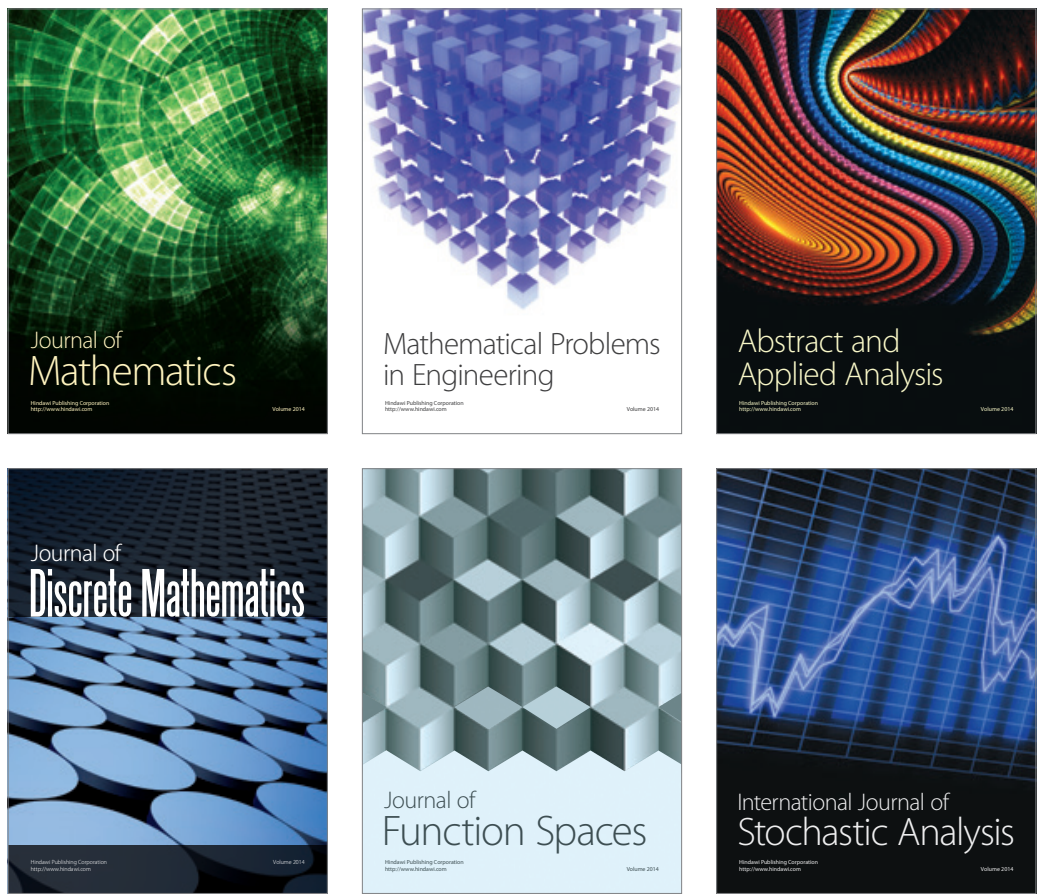

Journal of

Function Spaces

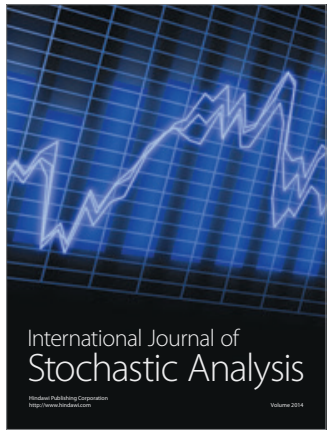

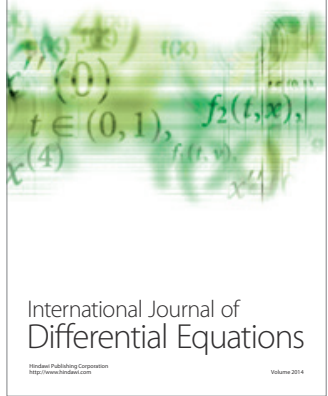
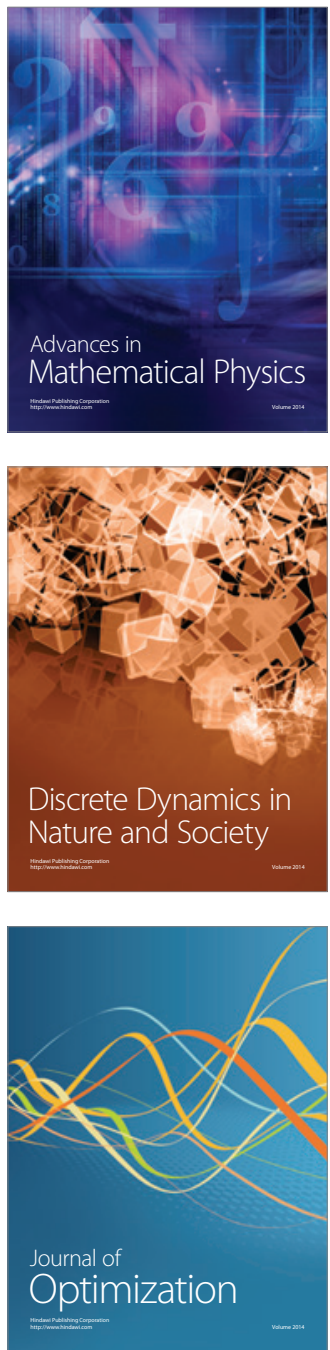